\title{
Cardiac rehabilitation protocols outcome in frail patients undergoing transcatheter aortic valve implantation
}

\author{
Mihaela Mocan ${ }^{1,2}$, Roxana Chiorescu ${ }^{1}$, Oana Natalia Banc ${ }^{2}$, Bogdan Mocan ${ }^{3}$, Florin Anton ${ }^{1,4}$, Mirela Stoia ${ }^{1,4}$, Anca \\ Daniela Farcas ${ }^{1,4}$
}

Corresponding author: : Anton Florin Petru, E-mail address: florinantonfr@yahoo.com

1. Internal Medicine Department, "Iuliu Hatieganu" University of Medicine and Pharmacy, Cluj-Napoca 2. Internal Medicine Department, Emergency Clinical County Hospital, Cluj-Napoca

3. Design Engineering and Robotics, Technical University of Cluj-Napoca

4. Cardiology Department, Emergency Clinical County Hospital, Cluj-Napoca

\begin{abstract}
The degenerative aortic stenosis (AS) is the most frequent valvular disease in the elderly (over 75 years), and its severe form is found in $12.4 \%$ associated with a low survival rate at 5 years $(15-50 \%)$. These patients are categorised as frail and the only possible choice of treatment is transcatheter aortic valve implantation (TAVI). When cardiac rehabilitation programs (CRP) were introduced after TAVI, they had a positive impact on clinical outcome, increasing the quality of life and decreasing morbidity and mortality, especially in the elderly with high frailty score.

This review underlines the utility of objective and functional frailty assessment in elderly patients with severe AS, before and after TAVI. Inclusion of these patients in complex and individually designed CRP could improve both QoL and short and long term outcome. Apart from exercise recommendation, a complete and ideal CRP should include nutrition counselling, occupational therapy, and psychological counselling to ensure psychosocial health, as well as social worker counselling.

CRP could prevent and reduce severity of frailty by improving/increasing mobility, muscle mass and cognitive function. Further studies should aim at appraising long-term effects and prognostic relevance of CRP in frail patients undergoing TAVI.

Key words: cardiac rehabilitation protocols, frailty assessment methods, severe aortic stenosis, transcatheter aortic valve implantation,
\end{abstract}

\section{Introduction}

In the last decades, we have witnessed a drastic increase in the proportion of elderly in the Western countries. Thus, in $2013,32 \%$ of deaths worldwide were caused by cardiovascular diseases [1]. Among these, the degenerative aortic stenosis (AS) is the most frequent valvular disease in the elderly (over 75 years), and its severe form is found in $12.4 \%$ associated with a low survival rate at 5 years $(15-$ $50 \%$ ) [2]. Currently, there is no effective medical treatment available for these patients, and classic valve replacement surgery is prohibited in more than $30 \%$ of the cases due to excessive operative risk, advanced age or severe comorbidities [3], [4]. These patients have frailty i.e. "a biological syndrome that reflects a state of decreased physiological reserve and vulnerability to stressors such as chronical illness or cardiac surgery" [5]. Frailty is a high-priority subject in cardiovascular medicine due to aging and increasingly complex nature of the patients. The frailty context has pushed science further and new devices and procedures have been developed to enable clinicians to treat a wider array of patients [6]. For the frail patients with severe and symptomatic AS, the only possible choice of treatment is transcatheter aortic valve implantation (TAVI).
In the past decade, over 100,000 patients worldwide underwent TAVI. The choice of TAVI against surgically aortic valve replacement (sAVR) was driven by increasing evidence of better outcome in frail patients, as showed in the PARTNER-B study [7]. Moreover, when cardiac rehabilitation programs (CRP) were introduced after TAVI, they had a positive impact on clinical outcome, increasing the quality of life and decreasing morbidity and mortality, especially in the elderly with high frailty score [6].

Thus, the present article aims at reviewing the means of assessing frailty and the benefits of cardiac rehabilitation in frail patients who undergone TAVI.

\section{Frailty assessment methods}

Frailty is recognized as a biological syndrome, present in about $5 \%$ of the population aged 60 and over, which predicts the risk of adverse outcomes [8]. It is associated with a multisystem decline in the physiological reserve and increased vulnerability to stressors, resulting in an increased risk of adverse outcomes such as disability, hospitalization and death [5]. There is no consensus regarding frailty assessment. Many different ways to evaluate frailty have been reported, each scale evaluating different domains: deficit accumulation, function and/or 
biology [9]. The Frailty Index (FI) has been developed on the concept of deficit accumulation - a combination of symptoms, diseases, conditions and disability [10 ], while the biological model known as the Cardiovascular Health Study (CHS) scale is based on five components - weight loss, exhaustion, low energy expenditure [11]. FI results could be improved by adding Mini Mental State Examination (MMSE), Mini Nutritional Assessment (MNA), Activities of Daily Living (ADL), Instrumental Activities of Daily Living (IADL), Timed Up and Go (TUG) and a subjective mobility disability (defined as a decreased frequency of walking $200 \mathrm{~m}$ and/or of climbing stairs). Both CHS and FI models are difficult to use in clinical practice because they require performance measurement (for CHS - walking speed, adhesion, strength, and more than 40 components for the FI, for example: knowledge, performance, physical etc.) [12]. Another scale proposed in the study of osteoporotic fractures - the SOF scale, - includes a functional factor and two biological factors : - the inability to lift five times from a chair without the use of arms, weight loss and low energy [13]. Although it requires measurement of performance, it is short and easy to operate with it in the clinic. The FRAIL scale is a combination of functional, deficit accumulation, and biological frailty models [14]. It requires a minimal administration time, and it can be easily used in clinical practice and can predicts negative health outcomes. Frail patients also have a poor life quality as evaluated by quality of life (QoL) questionnaire [15].

A large multicentric trial regarding frailty in old patients undergoing TAVI is FRAILTY-AVR [16]. It included 1020 patients (14 centers, 3 countries) evaluated by multiple frailty scales. The results showed that the prevalence of frailty ranged between $28 \%$ and $68 \%$, depending on the frailty scale. The frailty assessment added incremental value to classic pre- and post-operatory risk-scores. At one year after TAVI, more than $50 \%$ of patients initially categorized as frail suffered functional decline and had higher mortality rates. Another study derived from the OCEAN-TAVI Japanese multicenter registry investigated the 5 gait speed $(\mathrm{m} / \mathrm{sec})$ in 1256 patients who underwent TAVI. They proved that gait speed is an independent negative prognosticis marker, correlating with increased mortality after the procedure.

\section{Assessment methosds of the cardiac rehabilitation protocols outcome (advantages and disadvantages)}

Various assessment methods of CRP were studied in literature. They are divided in three great domains: exercise tolerance evaluation, functional independence and QoL measurements. The exercise tolerance may be evaluated by 6 minutes waking test $(6 M W T)$, by functional impedance or by maximal exercise capacity (work load). 6MWT is a simple functional test known to correlate significantly with NYHA class, quality of life and mortality, especially in advanced heart failure patients [17]. An interesting, positive correlation was found between 6MWT performance and BMI higher than $30 \mathrm{~kg} / \mathrm{m}^{2}$ [18]. This correlation, even paradoxical, is explained by our previous results showing that obese patients often have a better outcome, even though they are more prone cu to cardiac dysfunction and cardiovascular diseases [19], [20]. Maximal exercise capacity or physical capacity may be evaluated by using cardiopulmonary exercise testing (CPET) with a cycle ergometer following standardised protocols, as described recently by Sibilitz et al [21].

For functional independence evaluation the Barthel Index (BI) is frequently used. BI is an autonomy index. That uses a scale of $0-100$ to rate the degree of independence in activities of daily living, where 0 is total dependence and 100 is total independence [22]. As an alternative, functional independence measure (FIM) assesses physical and cognitive disability and encompasses several items necessary for daily function [23]. The score ranges between 18 and 126, with higher scores indicating more independence. In the same range, the hospital and anxiety depression scale $(H A D S)$ is a fourteen item scale with seven items relating to anxiety and seven to depression, with higher numbers indicating more distress.

Quality of life may be assessed using QoL scales, as described previously [15]. Examples of such scales are Kansas City Cardiomyopathy Questionnaire (KCCQ) and the Medical Outcomes Study 12-Item Short-Form Health Survey (SF-12). The KCCQ is a 23-item, self-administered questionnaire evaluating physical and social function, and symptom severity. The SF-12 consists of 12 questions referring to different aspects of physical and mental function and generating 2 summary scores [24]. 
Cardiac rehabilitation protocols recommended after TAVI and the effect on outcome

Many international rehabilitation societies such as The International British and Canadian Associations for Cardiovascular Prevention and Rehabilitation, American Association of Cardiovascular and Pulmonary Rehabilitation, or the European Association of Preventive Cardiology, have established guidelines to ensure a benefit from CRP [25]. There are general recommendations regarding:

1. Aerobic exercise, outdoor walking, treadmill exercises or riding a stationary bicycle (30-60 minutes, 3-5 times per week aiming a $50 \%-80 \%$ of the HR achieved in exercise testing);

2. Dynamic resistance exercise using progressive elastic bands (2-3 times per week, intensity of 1015 repetitions, 1-3 sets for upper and lower extremities);

3. Isometric exercise is generally not recommended, because it might increase the blood pressure [25].

ACC 2017 Guidelines recommend early mobilization, immediately after TAVI and "cardiac rehabilitation and promotion of physical activity as appropriate", on long term follow-up [26]. European guidelines recommend (Class IB) that post valve surgery exercise training can be started early inhospital, with CRP consisting of "exercise training individually tailored according to the baseline exercise capacity, ventricular function, and different valve surgery" and should last 2-4 weeks for inpatient or up to 12 weeks for out-patient settings clinical condition [27].

An elegant meta-analysis published recently by Tamuleviciute-Prasciene E. et al [28] evaluated 2623 articles in valvular patients, among which 61 focused on frailty and only 12 on CRP with exercise-based protocols (??) clearly described in the methods. Some of the studies were carried out in order to assess the safety and the beneficial effects of various types of CRP protocols in patients undergoing TAVI [7], [29], [30], [31], [18]. According to TamuleviciutePrasciene E. et al [18] different exercise training programs applied in elderly patients after surgery/intervention were safe and effective in improving functional and physical capacity, QoL and independence in daily life activities. In frail patients with severe AS, before and after TAVI, exercisebased programs increased gait speed and improved balance and performance in activities of daily life [28]. In conclusion, after this vast literature review, apart from exercise recommendation, a complete and ideal CRP should include nutrition counselling, occupational therapy, and psychological counselling to ensure psychosocial health, and social worker counselling [32]. As for the settings of CRP, beside specialised center supervision, smart homes and distance monitored CRP could be implemented, lowering the cost of the procedures [33].

CRP proved to be a safe and an effective therapeutic choice for the patients undergoing TAVI, regardless the type of exercise recommended [31]. For example, Fauchere et al. in a retrospective study, evaluated a CRP consisting of "supervised gymnastics, aerobic and respiratory workout sessions" of a low/medium intensity, 2- 3 times per day, 6 days per week [34]. Even though, the patients that undergone TAVI were older and sicker than the patients that undergone surgery, they have similar benefits assessed by FIM, 6MWT and HADS [34]. Later, Russo et al tested another CPR consisting of "30 min of respiratory workout, followed by an aerobic session on a cyclette in the morning and, in the afternoon, $30 \mathrm{~min}$ of callisthenic exercises", of a low/medium intensity, 6 days per week [35]. The outcome was evaluated using 6MWT, BI and CPET. The results showed that professional supervised CRP enhances independence, mobility and functional capacity and should be encouraged in all patients.

Eicher et al. conducted a prospective cohort multicentric study on 136 patients who were referred to multicomponent three-week cardiac rehabilitation programme in one of the three participating cardiac rehabilitation centers. Patients were stratified into groups according to maximal exercise capacity at initial exercise testing: $<1.0 \mathrm{~W} / \mathrm{kg}$ and $>1.0 \mathrm{~W} / \mathrm{kg}$. The CRP consisted of ergometer endurance training five times per week, average bicycle training sessions lasting about $30 \mathrm{~min}$. Patients with a higher physical capacity $(>1.0 \mathrm{~W} / \mathrm{kg})$ were recommended additional strength training at $30-50 \%$ at their one repetition maximum on common weigh machine. When considered appropriate, outdoor walking, aqua or spinal gymnastics, in groups were added to physical therapy [18]. As a result of CRP the number of frail patients was significantly reduced by $9 \%$ (from $36.9 \%$ to $27.9 \%$ ). The overall FI decreased by 0.4 points, and significant changes in cognition parameters (MMSE), nutrition (MNA), and subjective mobility disability and mobility (TUG) were observed. QoL improved significantly and 
anxiety was decreased. None of the quality parameters influenced the prognostic.

\section{Conclusion}

This review underlines the utility of objective and functional frailty assessment in elder patients with severe AS, before and after TAVI. Inclusion of these patients into complex and individually designed CRP could improve both QoL and short and long term outcome. CRP could prevent and reduce severity of frailty by improving mobility, muscle mass and cognitive function. Further studies should aim at appraising long-term effects and prognostic relevance of CRP in frail patients undergoing TAVI.

\section{References}

1. Roth GA, Huffman MD, Moran AE, Feigin V, Mensah GA, Naghavi M, et al. Global and regional patterns in cardiovascular mortality from 1990 to 2013. Circulation 2015;132:1667-78. doi:10.1161/CIRCULATIONAHA.114.008720.

2. Osnabrugge RLJ, Mylotte D, Head SJ, Van Mieghem NM, Nkomo VT, Lereun CM, et al. Aortic stenosis in the elderly: Disease prevalence and number of candidates for transcatheter aortic valve replacement: A meta-analysis and modeling study. J Am Coll Cardiol 2013;62:1002-12. doi:10.1016/j.jacc.2013.05.015.

3. Vahanian A, Alfieri O, Andreotti $\mathrm{F}$, Antunes MJ, Barón-Esquivias G, Baumgartner H, et al. Guidelines on the management of valvular heart disease (version 2012). Eur Heart J 2012;33:2451-96. doi:10.1093/eurheartj/ehs109.

4. Pereira E, Silva G, Caeiro D, Fonseca M. What has changed in surgical treatment of severe aortic stenosis with the advent of percutaneous intervention? Rev Port Cardiol 2013;32:749-56.

5. Afilalo J, Alexander KP, Mack MJ, Maurer MS, Green P, Allen LA, et al. Frailty assessment in the cardiovascular care of older adults. J Am Coll Cardiol 2014;63:747-62. doi:10.1016/j.jacc.2013.09.070.

6. Singh M, Stewart R, White H. Importance of frailty in patients with cardiovascular disease. Eur Heart J 2014;35:1726-31. doi:10.1093/eurheartj/ehu197.

7. Reynolds M, Magnuson E, Wang K, Lei Y. CostEffectiveness of Transcatheter Aortic Valve Replacement Compared With Standard Care Among Inoperable Patients With Severe Aortic StenosisClinical. Circulation 2012;125:1102-9. doi:10.1161/CIRCULATIONAHA.111.054072.

8. Wilhelm-Leen E, Hall Y, Tamura M et al. Frailty and chronic kidney disease: The Third National Health and Nutrition Evaluation Survey. Am J Med 2009;122:664-71.
9. Wong C, Weiss D, Sourial $\mathrm{N}$ et al. Frailty and its association with disability and comorbidity in a community-dwelling sample of seniors in Montreal: A cross-sectional study. Aging Clin Exp Res 2010:54-62.

10. Rockwood K MA. Frailty in relation to the accumulation of deficits. J Gerontol A Biol Sci Med Sci 2007;62A:722-727.

11. Fried L, Tangen C, Walston $\mathrm{J}$ et al. Cardiovascular Health Study Collaborative Research Group. Frailty in older adults: Evidence for a phenotype. J Gerontol A Biol Sci Med Sci 2001;56A:M146-M156.

12. Malmstrom T, Miller D, Morley J. A Comparison of Four Frailty Models. J Am Geriatr Soc 2014;62:7216.

13. Cawthon P, Marshall L, Michael Y et. Osteoporotic Fractures in Men Research Group. Frailty in older men: Prevalence, progression, and relationship with mortality. J Am Geriatr Soc 2007;55:1216-1223.

14. Abellan van Kan G, Rolland Y, Bergman $\mathrm{H}$ et al. The I.A.N.A. Task Force on frailty assessment of older people in clinical practice. J Nutr Heal Aging 2008;12:29-37.

15. Farcaş AD, Năstasă LE, Anton FP, Stoia MA, Goidescu M, Mocan Hognogi DL, Mocan M, Vonica CL, Vida-Simiti L. Quality of life - an important parameter of cardiac rehabilitation in heart failure patients. Balneo Res J 2018;9:288-90.

16. Afilalo J, Lauck S, Kim DH, Lefèvre T, Piazza N, Lachapelle K, et al. Frailty in Older Adults Undergoing Aortic Valve Replacement: The FRAILTY-AVR Study. J Am Coll Cardiol 2017;70:689-700. doi:10.1016/j.jacc.2017.06.024.

17. Opasich C, De Feo S, Pinna GD, Furgi G, Pedretti R, Scrutinio D, et al. Distance walked in the 6-minute test soon after cardiac surgery: Toward an efficient use in the individual patient. Chest 2004;126:1796801. doi:10.1378/chest.126.6.1796.

18. Eichler S, Salzwedel A, Reibis R, Nothroff J, Harnath A, Schikora M, et al. Multicomponent cardiac rehabilitation in patients after transcatheter aortic valve implantation: Predictors of functional and psychocognitive recovery. Eur J Prev Cardiol 2017;24:257-64. doi:10.1177/2047487316679527.

19. Alexescu TG, Cozma A, Sitar-Tăut A, Negrean V, Handru MI, Motocu M, et al. Cardiac Changes in Overweight and Obese Patients. Rom J Intern Med 2016;54:161-72. doi:10.1515/rjim-2016-0022.

20. Mocan M, Anton F, Suciu Š, Răhian R, Blaga SN, Fărcaş AD. Multimarker Assessment of Diastolic Dysfunction in Metabolic Syndrome Patients. Metab Syndr Relat Disord 2017;15:507-14. doi:10.1089/met.2017.0060.

21. Sibilitz KL, Berg SK, Rasmussen TB, Risom SS, Thygesen LC, Tang L, et al. Cardiac rehabilitation 
increases physical capacity but not mental health after heart valve surgery: A randomised clinical trial. Heart 2016;102:1995-2003. doi:10.1136/heartjnl-2016309414 .

22. Sainsbury A, Seebass G, Bansal A, Young JB. Reliability of the Barthel Index when used with older people. Age Ageing 2005;34:228-32. doi:10.1093/ageing/afi063.

23. Cournan M. Use ofthe functional independencemeasure for outcomesmeasurement in acute inpatient rehabilitation. Rehabil Nurs 2011;36:111-1.

24. Pressler A, Christle JW, Lechner B, Grabs V, Haller B, Hettich I, et al. Exercise training improves exercise capacity and quality of life after transcatheter aortic valve implantation: A randomized pilot trial. Am Heart $\mathrm{J}$ 2016;182:44-53. doi:10.1016/j.ahj.2016.08.007.

25. Pesah E, Supervia M, Turk-Adawi K, Grace SL. A Review of Cardiac Rehabilitation Delivery Around the World. Prog Cardiovasc Dis 2017;60:267-80. doi:10.1016/j.pcad.2017.08.007.

26. Otto CM, Kumbhani DJ, Alexander KP, Calhoon JH, Desai MY, Kaul S, et al. 2017 ACC Expert Consensus Decision Pathway for Transcatheter Aortic Valve Replacement in the Management of Adults With Aortic Stenosis. J Am Coll Cardiol 2017;69:1313-46. doi:10.1016/j.jacc.2016.12.006.

27. Corr U, Carré F, Heuschmann P, Hoffmann U, Verschuren M, Halcox J, et al. Secondary prevention through cardiac rehabilitation: Physical activity counselling and exercise training. Eur Heart J 2010;31:1967-76. doi:10.1093/eurheartj/ehq236.

28. Tamuleviciute-Prasciene E, Drulyte K, Jurenaite G, Kubilius R, Bjarnason-Wehrens B. Frailty and Exercise Training: How to Provide Best Care after Cardiac Surgery or Intervention for Elder Patients with Valvular Heart Disease. Biomed Res Int 2018;2018:1-36. doi:10.1155/2018/9849475.

29. Genta FT, Tidu M, Bouslenko Z, Bertolin F, Salvetti I, Comazzi F, et al. Cardiac rehabilitation after transcatheter aortic valve implantation compared to patients after valve replacement. J Cardiovasc Med 2017;18:114-20. doi:10.2459/JCM.0000000000000494.

30. Voller H, Salzwedel A, Nitardy A, Buhlert H. Effect of cardiac rehabilitation on functional and emotional status in patients after transcatheter aortic-valve implantation. Eur J Prev Cardiol 2014;22:568-74. doi:10.1177/2047487314526072.

31. Ribeiro GS, Melo RD, Deresz LF, Dal Lago P, Pontes MRN, Karsten M. Cardiac rehabilitation programme after transcatheter aortic valve implantation versus surgical aortic valve replacement: Systematic review and meta-analysis. Eur J Prev Cardiol 2017;24:68897. doi: $10.1177 / 2047487316686442$.

32. Farcaş AD, Năstasă LE. Factors influencing the perception of stress in patients with heart failure. Procedia - Social and Behavioral Sciences 127 ( 2014 ) $144-148$

33. Sitar-Taut A, Sitar-Taut-Dan-Andrei, Cramariuc O, Negrean V, Sampelean D, Rusu L, Orăşan O, Fodor A., Dogaru G, Cozma A. Smart homes for older people involved inrehabilitation activities - reality or dream, acceptance or rejection?, Balneo Research Journal 2018; 9:291-8. doi:http://dx.doi.org/10.12680/balneo.2018.199.

34. Fauchère I, Weber D, Maier W, Altwegg L, Lüscher $\mathrm{TF}$, Grünenfelder J, et al. Rehabilitation after TAVI compared to surgical aortic valve replacement. Int J Cardiol 2014;173:564-6. doi:10.1016/j.ijcard.2014.03.121.

35. Russo N, Compostella L, Tarantini G, Setzu T, Napodano M, Bottio T, et al. Cardiac rehabilitation after transcatheter versus surgical prosthetic valve implantation for aortic 